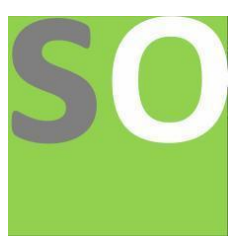

Article title: A Machine-Level Interpretation of Deductive Rules on Enact I and Enact II Model Theorems.

Authors: Frank Appiah[1]

Affiliations: UCL CENTRE OF SYSTEMS ENGINEERING[1]

Orcid ids: 0000-0001-6732-9503[1]

Contact e-mail: appiahnsiahfrank@gmail.com

License information: This work has been published open access under Creative Commons Attribution License $\mathrm{http}: / / c r e a t i v e c o m m o n s . o r g / l i c e n s e s / b y / 4.0 /$, which permits unrestricted use, distribution, and reproduction in any medium, provided the original work is properly cited. Conditions, terms of use and publishing policy can be found at https://www.scienceopen.com/.

Preprint statement: This article is a preprint and has not been peer-reviewed, under consideration and submitted to ScienceOpen Preprints for open peer review.

Funder: RF/202021/20/222

DOI: 10.14293/S2199-1006.1.SOR-.PPVOHXY.v1

Preprint first posted online: 05 October 2020

Keywords: model, composites, enactment, logic, proof, syntactic, theorem, enactprover, machine, Program 


\title{
A Machine-Level Interpretation of Deductive Rules on Enact I and Enact II Model Theorems. (ENACTPROVER)
}

\author{
FRANK APPIAH ${ }^{1}$. \\ UNIVERSITY COLLEGE LONDON, FACULTY OF ENGINEERING SCIENCE, CENTRE \\ OF SYSTEMS ENGINEERING, GLOWER, ENGLAND, UNITED KINGDOM. \\ frank.appiah@ucl.ac.uk \\ appiahnsiahfrank@gmail.com. \\ Extended Abstract ${ }^{+}$. This research proposal is on provable forms based on \\ syntactic theorem using Kleene Axiom schema. Enact model I and II of \\ propositional formulas from enactment logic are proven in terms of theorems \\ based on deductive rules. Work proves by deduction rules that Enact Model I \\ and II are model theorems[1] in machine-level interpretation. Enactprover is a \\ machine program for reading and writing Kleene theorem proving axioms based \\ on enactment logic.
}

Keywords. model, composites, enactment, logic, proof, syntactic, theorem. enactprover, machine-interpretation, program.

Year of award: 2020

Year of Publication: 2020

1 AFFILIATE. UNIVERSITY OF LONDON, KING'S COLLEGE LONDON, DEPARTMENT OF ENGINEERING, LONDON, UK.

This person is awarded research fellowships, 22 September, 2020- Engineering for Development Research Fellowship for 5 years. 


\section{INTRODUCTION}

This research is based on axiomatical schemas[3] of Kleene that gives theorems based on proofs of enactment models[1]. Syntactic computing[3] in the propositional calculus is based on using axioms and deduction rules[3] to produce theorems. Kleene[3] give the following axiom schemas:

(1a) $\alpha \rightarrow(\beta \rightarrow \alpha)$.

(1b) $(\alpha \rightarrow \beta) \rightarrow(\alpha \rightarrow(\beta \rightarrow \gamma) \rightarrow(\alpha \rightarrow \gamma))$.

(2a) $\alpha \rightarrow(\beta \rightarrow(\alpha \wedge \beta))$,

(2b) $(\alpha \rightarrow \beta) \rightarrow \alpha$.

(2c) $(\alpha \wedge \beta) \rightarrow \beta$.

(3a) $\alpha \rightarrow(\alpha \vee \beta)$,

(3b) $\beta \rightarrow(\alpha \vee \beta)$,

(3c) $\quad(\alpha \rightarrow \gamma) \rightarrow((\beta \rightarrow \gamma) \rightarrow((\alpha \vee \beta) \rightarrow \gamma))$.

(4a) $(\alpha \rightarrow \beta) \rightarrow((\alpha \rightarrow \neg \beta) \rightarrow \neg \alpha)$,

(4b) $(\neg \neg \alpha) \rightarrow \alpha$.

\section{Results of Work[1]:}

The models of enactment logic are:
(1) enact $_{E} \rightarrow$ enact $_{L}$
(2) $\quad a_{i} \rightarrow a$
(3) $a_{j} \rightarrow l$
(4) $\operatorname{rank}_{i} \rightarrow t$
(5) enact $_{E} \rightarrow\left(\right.$ enact $\left._{E} \rightarrow a_{i}\right)$ 

(6) enact $_{L} \rightarrow\left(a_{j} \rightarrow l\right)$
(7) $\quad a_{i} \rightarrow\left(a_{j} \rightarrow l\right)$
(8) $a_{j} \rightarrow\left(l \rightarrow \operatorname{rank}_{i}\right)$
(9) $\quad l \rightarrow\left(\operatorname{rank}_{i} \rightarrow t\right)$

Axiom is produced by replacing the Greek variablee of an axiom schema by formula. Models (5) and (6) will be proved using Kleene expression axioms (1a) to $(4 b)$.

\section{THEOREM PROVING.}

Let Enact $_{E}$ to be represented by $E_{E}$.

Let Enact $_{L}$ to be represented by $E_{L}$.

(5) enact $_{E} \rightarrow\left(\right.$ enact $\left._{E} \rightarrow a_{i}\right)$ : Enact Model I

(5) is proven by theorem 5 as shown below:

\section{Theorem 5:}

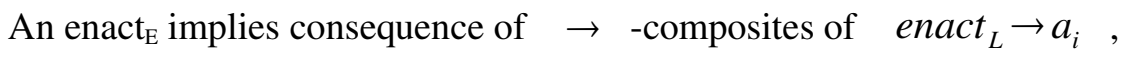
expressed as: enact $_{E} \rightarrow\left(\right.$ enact $\left._{E} \rightarrow a_{i}\right)$, then it is the consequence of $\left(\left(\right.\right.$ enact $_{E} \rightarrow$ enact $\left._{L}\right) \rightarrow\left(\right.$ enact $\left.\left._{E} \rightarrow a_{i}\right)\right)$.

Proof. Theorem 5 is expressed as $\quad\left(\left(E_{E} \rightarrow E_{L}\right) \rightarrow\left(E_{E} \rightarrow a_{i}\right)\right)$. 
Axiom. (1) $\left(\left(E_{E} \rightarrow E_{L}\right) \rightarrow\left(E_{E} \rightarrow a_{i}\right)\right)$. given can be proved from

Kleene's Axiom.

(2) $\left(\left(E_{E} \rightarrow\left(E_{L} \rightarrow a_{i}\right)\right) \rightarrow\left(E_{E} \rightarrow E_{L}\right) \rightarrow\left(E_{E} \rightarrow\left(E_{L} \rightarrow a_{i}\right)\right)\right)$, axiom of schema (1a).

(3) $\quad\left(\left(E_{E} \rightarrow E_{L}\right) \rightarrow\left(E_{E} \rightarrow\left(E_{L} \rightarrow a_{i}\right)\right)\right)$ modus ponens of lines 1 and 2 .

Deduction Rule. $\quad \frac{\alpha,(\alpha \rightarrow \beta)}{\beta}$, where $\beta$ is a consequence of $\alpha$ and $(\alpha \rightarrow \beta)$

$$
\begin{aligned}
& \frac{\left(E_{E} \rightarrow\left(E_{L} \rightarrow a_{i}\right)\right)=\alpha, \quad\left(\alpha \rightarrow\left(E_{E} \rightarrow E_{L}\right) \rightarrow\left(E_{E} \rightarrow\left(E_{L} \rightarrow a_{i}\right)\right)=\beta\right)}{\beta} \\
& \left(E_{E} \rightarrow E_{L}\right) \rightarrow\left(E_{E} \rightarrow\left(E_{L} \rightarrow a_{i}\right)\right)=\beta \text { is the consequence of } \\
& \left(E_{E} \rightarrow\left(E_{L} \rightarrow a_{i}\right)\right)=\alpha \text { and } \quad\left(E_{E} \rightarrow E_{L}\right) \rightarrow\left(E_{E} \rightarrow\left(E_{L} \rightarrow a_{i}\right)\right)=\beta .
\end{aligned}
$$

(4) $\begin{aligned} & \left(\left(E_{E} \rightarrow E_{L}\right) \rightarrow\left(E_{E} \rightarrow\left(E_{L} \rightarrow a_{i}\right)\right)\right) \\ & \rightarrow\left(\left(\left(E_{E} \rightarrow E_{L}\right) \rightarrow\left(\left(E_{E} \rightarrow\left(E_{L} \rightarrow a_{i}\right)\right) \rightarrow\left(E_{E} \rightarrow a_{i}\right)\right)\right) \rightarrow\left(\left(E_{E} \rightarrow E_{L}\right) \rightarrow\left(E_{E} \rightarrow a_{i}\right)\right)\right)\end{aligned}$

axiom of schema (1b).

(5) $\quad\left(\left(E_{E} \rightarrow E_{L}\right) \rightarrow\left(\left(E_{E} \rightarrow\left(E_{L} \rightarrow a_{i}\right)\right) \rightarrow\left(E_{E} \rightarrow a_{i}\right)\right)\right) \rightarrow\left(\left(E_{E} \rightarrow E_{L}\right) \rightarrow\left(E_{E} \rightarrow a_{i}\right)\right)$

modus ponens of lines 3 and 4 .

Deduction Rule. $\frac{\alpha,(\alpha \rightarrow \beta)}{\beta}$, modus ponens 3 and 4 gives,

$$
\frac{\alpha_{4}=\alpha_{3,},\left(\alpha_{4}=\alpha_{3} \rightarrow \beta_{4}\right)}{\beta_{4}} .
$$

$\beta_{4}$ is a consequence of $\alpha_{3}=\alpha_{4}$ and $\left(\alpha_{3}=\alpha_{4} \rightarrow \beta_{4}\right)$.

$$
\frac{\left(E_{E} \rightarrow E_{L}\right) \rightarrow\left(E_{E} \rightarrow\left(E_{L} \rightarrow a_{i}\right)\right)=\alpha_{3}=\alpha_{4},\left(\alpha_{3}=\alpha_{4} \rightarrow, \beta_{4}\right)}{\left(\left(E_{E} \rightarrow E_{L}\right) \rightarrow\left(\left(E_{E} \rightarrow\left(E_{L} \rightarrow a_{i}\right)\right) \rightarrow\left(E_{E} \rightarrow a_{i}\right)\right)\right) \rightarrow\left(\left(E_{E} \rightarrow E_{L}\right) \rightarrow\left(E_{E} \rightarrow a_{i}\right)\right)=\beta_{4}}
$$


(6) An axiom of schema (1b) gives,

$$
\left(E_{E} \rightarrow E_{L}\right) \rightarrow\left(\left(E_{E} \rightarrow\left(E_{L} \rightarrow a_{i}\right)\right) \rightarrow\left(E_{E} \rightarrow a_{i}\right)\right) .
$$

(7) Modus ponens on line 5 and 6 gives,

$$
\begin{gathered}
\frac{\alpha_{5}=\alpha_{6,}\left(\alpha_{5}=\alpha_{6} \rightarrow \beta_{5}\right)}{\beta_{5}} \\
\frac{\left(E_{E} \rightarrow E_{L}\right) \rightarrow\left(\left(E_{E} \rightarrow\left(E_{L} \rightarrow a_{i}\right)\right) \rightarrow\left(E_{E} \rightarrow a_{i}\right)\right)=\alpha_{5}=\alpha_{6}\left(\alpha_{5}=\alpha_{6} \rightarrow \beta_{5}\right)}{\left(\left(E_{E} \rightarrow E_{L}\right) \rightarrow\left(E_{E} \rightarrow a_{i}\right)\right)=\beta_{5}}
\end{gathered}
$$

$\beta_{5}$ is the consequence of $\alpha_{5}=\alpha_{6}$ and $\left(\alpha_{5}=\alpha_{6} \rightarrow \beta_{5}\right)$. Thus

$$
\begin{aligned}
& \left(E_{E} \rightarrow E_{L}\right) \rightarrow\left(E_{E} \rightarrow a_{i}\right) \text {. Hence } \\
& E_{E} \rightarrow\left(E_{L} \rightarrow E_{L}\right) \mid-\left(E_{E} \rightarrow E_{L}\right) \rightarrow\left(E_{E} \rightarrow a_{i}\right) . \\
& E_{E}, E_{L}, E_{L} \mid-\left(E_{E} \rightarrow E_{L}\right) \rightarrow\left(E_{E} \rightarrow a_{i}\right)
\end{aligned}
$$

$\left(E_{E} \rightarrow E_{L}\right) \rightarrow\left(E_{E} \rightarrow a_{i}\right)$ is a theorem.

\section{(6) enact $_{L} \rightarrow\left(a_{j} \rightarrow l\right)$ : Enact Model II}

(6) is proven by theorem 6 as shown below:

\section{Theorem 6:}

An enact implies consequence of $\rightarrow$-composites of $a_{j} \rightarrow l$, expressed as: enact $_{L} \rightarrow\left(a_{j} \rightarrow l\right)$, then it is the consequence of

$$
\left(\left(\text { enact }_{L} \rightarrow a_{j}\right) \rightarrow\left(\text { enact }_{L} \rightarrow l\right)\right) .
$$

Proof. Theorem 6 is expressed as $\quad\left(\left(E_{L} \rightarrow a_{j}\right) \rightarrow\left(E_{L} \rightarrow l\right)\right)$. 
Axiom. (1) $\left(\left(E_{L} \rightarrow a_{j}\right) \rightarrow\left(E_{L} \rightarrow l\right)\right)$. given can be proved from Kleene's

Axiom.

(2) $\quad\left(\left(E_{L} \rightarrow\left(a_{j} \rightarrow l\right)\right) \rightarrow\left(E_{L} \rightarrow a_{j}\right) \rightarrow\left(E_{L} \rightarrow\left(a_{j} \rightarrow l\right)\right)\right)$, axiom of schema (1a).

(3) $\quad\left(\left(E_{L} \rightarrow a_{j}\right) \rightarrow\left(E_{L} \rightarrow\left(a_{j} \rightarrow l\right)\right)\right)$ modus ponens of lines 1 and 2 .

Deduction Rule. $\quad \frac{\alpha,(\alpha \rightarrow \beta)}{\beta}$, where $\beta$ is a consequence of $\alpha$ and $(\alpha \rightarrow \beta)$.

$$
\frac{\left(E_{L} \rightarrow\left(a_{j} \rightarrow l\right)\right)=\alpha, \quad\left(\alpha \rightarrow\left(E_{L} \rightarrow a_{j}\right) \rightarrow\left(E_{L} \rightarrow\left(a_{j} \rightarrow l\right)\right)=\beta\right)}{\beta}
$$

$\left(E_{L} \rightarrow a_{j}\right) \rightarrow\left(E_{L} \rightarrow\left(a_{j} \rightarrow l\right)\right)=\beta \quad$ is the consequence of

$\left(E_{L} \rightarrow\left(a_{j} \rightarrow l\right)\right)=\alpha \quad$ and $\quad\left(E_{L} \rightarrow a_{j}\right) \rightarrow\left(E_{L} \rightarrow\left(a_{j} \rightarrow l\right)\right)=\beta \quad$

(4) $\begin{aligned} & \left(\left(E_{L} \rightarrow a_{j}\right) \rightarrow\left(E_{L} \rightarrow\left(a_{j} \rightarrow l\right)\right)\right) \\ & \rightarrow\left(\left(\left(E_{L} \rightarrow a_{j}\right) \rightarrow\left(\left(E_{L} \rightarrow\left(a_{j} \rightarrow l\right)\right) \rightarrow\left(E_{L} \rightarrow l\right)\right)\right) \rightarrow\left(\left(E_{L} \rightarrow a_{j}\right) \rightarrow\left(E_{L} \rightarrow l\right)\right)\right)\end{aligned}$

axiom of schema (1b).

(5) $\quad\left(\left(E_{L} \rightarrow a_{j}\right) \rightarrow\left(\left(E_{L} \rightarrow\left(a_{j} \rightarrow l\right)\right) \rightarrow\left(E_{L} \rightarrow l\right)\right)\right) \rightarrow\left(\left(E_{L} \rightarrow a_{j}\right) \rightarrow\left(E_{L} \rightarrow l\right)\right)$

modus ponens of lines 3 and 4 .

Deduction Rule. $\frac{\alpha,(\alpha \rightarrow \beta)}{\beta}$, modus ponens 3 and 4 gives,

$$
\frac{\alpha_{4}=\alpha_{3,}\left(\alpha_{4}=\alpha_{3} \rightarrow \beta_{4}\right)}{\beta_{4}} .
$$

$\beta_{4}$ is a consequence of $\alpha_{3}=\alpha_{4}$ and $\left(\alpha_{3}=\alpha_{4} \rightarrow \beta_{4}\right)$.

$$
\frac{\left(E_{L} \rightarrow a_{j}\right) \rightarrow\left(E_{L} \rightarrow\left(a_{j} \rightarrow l\right)\right)=\alpha_{3}=\alpha_{4},\left(\alpha_{3}=\alpha_{4} \rightarrow, \beta_{4}\right)}{\left(\left(E_{L} \rightarrow a_{j}\right) \rightarrow\left(\left(E_{L} \rightarrow\left(a_{j} \rightarrow l\right)\right) \rightarrow\left(E_{L} \rightarrow l\right)\right)\right) \rightarrow\left(\left(E_{L} \rightarrow a_{j}\right) \rightarrow\left(E_{E} \rightarrow l\right)\right)=\beta_{4}}
$$


(6) An axiom of schema (1b) gives,

$$
\left(E_{L} \rightarrow a_{j}\right) \rightarrow\left(\left(E_{L} \rightarrow\left(a_{j} \rightarrow l\right)\right) \rightarrow\left(E_{L} \rightarrow l\right)\right) .
$$

(7) Modus ponens on line 5 and 6 gives,

$$
\begin{gathered}
\frac{\alpha_{5}=\alpha_{6,}\left(\alpha_{5}=\alpha_{6} \rightarrow \beta_{5}\right)}{\beta_{5}} \\
\frac{\left(E_{L} \rightarrow a_{j}\right) \rightarrow\left(\left(E_{L} \rightarrow\left(a_{j} \rightarrow l\right)\right) \rightarrow\left(E_{L} \rightarrow a_{j}\right)\right)=\alpha_{5}=\alpha_{6,}\left(\alpha_{5}=\alpha_{6} \rightarrow \beta_{5}\right)}{\left(\left(E_{L} \rightarrow a_{j}\right) \rightarrow\left(E_{L} \rightarrow l\right)\right)=\beta_{5}}
\end{gathered}
$$

$\beta_{5}$ is the consequence of $\alpha_{5}=\alpha_{6}$ and $\left(\alpha_{5}=\alpha_{6} \rightarrow \beta_{5}\right)$. Thus $\left(E_{L} \rightarrow a_{j}\right) \rightarrow\left(E_{L} \rightarrow l\right)$.

Hence $E_{L} \rightarrow\left(a_{j} \rightarrow l\right) \mid-\left(E_{L} \rightarrow a_{j}\right) \rightarrow\left(E_{L} \rightarrow l\right)$.

$$
\begin{aligned}
& E_{L}, a_{j}, l \mid-\left(E_{L} \rightarrow a_{j}\right) \rightarrow\left(E_{L} \rightarrow l\right) \\
& \left(E_{L} \rightarrow a_{j}\right) \rightarrow\left(E_{L} \rightarrow l\right) \text { is a theorem. }
\end{aligned}
$$

The introduction is on enact model theorems and axioms developed from these models by Kleene axioms or theorems. The goals and objectives of this project is detailed below. Methodology to be used in the implementation approach and conduct of research are also discussed.

\section{ENACTPROVER: GOALS AND OBJECTIVES}

This research project has the following goals and objectives:

1. It is to implement an electronic machine provable solution capable of proving the model theorems of enactment logic. 
2. It is to interprets the parameters of enact function with enactprover interpreter.

3. It is to stream the outputs into an integrated development environment (Enactprover IDE) for display and human reading on communication.

4. Realistic passing values will be enacted to bring much meaning to the machine interpretation.

5. Novelty is in the programming style(color codes) of something of fellowship- something of colour.

6. Novelty again is in the graphical user interface theme in the development process.

The conduct of research will produce the following in such timelines:

\begin{tabular}{|l|c|}
\hline Conduct of Research & Timeline/years \\
\hline 1. Enactprover Interpreter & 1 \\
\hline 2. Graphical User Interface & 1 \\
\hline 3. Documentation & 2 \\
\hline 4. Final Dissemination & 1 \\
\hline Final Development & 5 \\
\hline
\end{tabular}

\section{METHODOLOGY}

This provides detail description of the exact work to be completed. The program work will indicate the research to be undertaken and the milestones that can be used to measure progress. The methodology to be used in this 
pursuit is looked at. The reason of choice of programming language is chosen to be $\mathrm{C}++$.

Both nationally and internationally, the author has already undertaken an intelligent system agent approach with semantic web and heuristics method in one Object-Oriented Deductive Reasoning Web software. Again, is an enact programming of semantic computation of enact function made by Enact Automated Reasoning with C++ system development. These projects were undertaken in UK locally and internationally. This current work is developed as follows:

$\mathrm{C}++$ interpreter methods are invoked from the passing enact parameters and the result of stream are displayed in a Java User Interface. This is a processor architecture based on command line interface in machine-mode application. The milestones are interpreter and automated office system called enact prover interpreter and automated enactprover system respectively.

\section{CONCLUSION AND FURTHERWORK}

This proposal is to develop an electronic machine in theorem proving of enactment logic. The challenge is to piece together a computing system based on Automated Enactprover System. This starts work on a deduction theorem proving on Enact model I and II of enactment logic in [1,2] by electronic program in a machine. Futherwork will discuss the three remaining enact models (III), (IV) and (V) of enactment logic. 
In such regard developing an electronically theorem proving machine for reading and writing of enactment logic is on headway in approachingly tackling the long write process.

\section{Compliance with Ethical Standards}

(In case of funding) Funding: This is research is funded mainly by Royal Academy of Engineering with reference detail: RF/202021/20/222. This is in collaboration with the University College London, Engineering Science, Centre of Systems Engineering where he is a professor since 2020.

\section{Conflict of Interest:}

Author, Prof. Frank Appiah declares that he has no conflict of interest .

\section{REFERENCES}

1. Appiah F. (2020). Syntactic Computation of Propositional Model Composites in Enactment Logic, KCL Art \& Science Research Office, Waterloo, England, United Kindgom.

2. Appiah F. (2020). Semantic Computation of Propositional Model Composites in Enactment Logic, KCL Art \& Science Research Office, Waterloo, England, United Kindgom.

3. Appiah F. (2009/10), RuleML for Policy Exchange in Agent Commerce, King's College London, Msc Dissertation.

4. Richard Stark W.(1990), Lisp, Lore, Logic, Springer Verlag, New York. 